\title{
A Double Layer Circularly Polarised Radial Line Slot Array Antenna with Uniform Aperture Illumination
}

\author{
Nishat Yasmin Koli ${ }^{1}$, Muhammad U. Afzal ${ }^{1}$, Karu P. Esselle ${ }^{2}$, Raheel M. Hashmi ${ }^{1}$, \\ Md Zahidul Islam ${ }^{3}$, and Sujan Shrestha ${ }^{1}$ \\ ${ }^{1}$ School of Engineering, Macquarie University, Sydney, NSW, Australia \\ ${ }^{2}$ School of Electrical and Data Engineering, University of Technology Sydney, Sydney, NSW, Australia \\ ${ }^{3}$ Teleaus: Serveno Australia Pty Ltd, Sydney, NSW, Australia \\ mst-nishat-yasmin.koli@students.mq.edu.au,muhammad.afzal@mq.edu.au
}

\begin{abstract}
In this paper, we have designed and investigated a double layer circularly polarised radial line slot array (RLSA) antenna for satellite communication. The antenna is composed of twofold radial waveguide with slots acting as radiating elements on its surface. The radiating slots are arranged in a spiral pattern on the antenna aperture. Every slot has a particular length and position. The slots are oriented in a way to intercept the radial currents on the upper waveguide. The slot lengths were varied to achieve a uniform aperture distribution. The electromagnetic power is fed from center of the lower waveguide. Numerical results show that the antenna is well matched within the operating frequency range. The far-field results indicate a peak directivity of $27 \mathrm{dBi}$ at $20 \mathrm{GHz}$ with a good pattern quality and lower side lobe level of $-27.2 \mathrm{~dB}$.
\end{abstract}

Index Terms-radial line slot array (RLSA), double layer waveguide, planar, low-profile, high directivity, satellite communication, good pattern quality

\section{INTRODUCTION}

The demand of satellite communication systems on moving platforms such as aircrafts, trains, buses, and ships are increasing day by day. Among the diversity of microwave and millimeter wave antennas, satellite communication commonly uses parabolic reflector antennas [1]. Despite of providing good coverage and efficiency, the use of parabolic reflectors on moving air, land, or sea vehicles are constrained by their bulky size, air resistance and snow build-up. Also its heavy mass is difficult for manufacturing and transportation and. The microstrip and phased array antennas are planar, provide high gain and easy to maintain but these antennas show low radiation efficiency at high gain [2]. Radial line slot array (RLSA) antennas are planar, low-weight and highly directives antennas. RLSA antennas act as an alternative to parabolic reflector and microstrip patch array antenna. Due to its high efficiency, lowprofile and high gain characteristics, RLSA antennas have been utilized for several years in satellite applications [1], [2]. The antennas utilise waveguide, which maintains outward travelling waves. These antennas have hundreds of slots on the antenna surface and act as radiating elements [3]. The RLSA antennas can be designed as circularly polarised, linearly polarised or elliptically polarised.

In this paper, we have designed and investigated the radiation performance of a double layer RLSA antenna. We have been investigating RLSA as a feed for our metasurface-based beamsteering technique [4]. The aim is to develop an antenna with a fixed broadside beam that has uniform phase distribution on the aperture and provides good pattern quality in its far-field. The paper is arranged such that the antenna design methodology is explained in Section II. Predicted near-field and far-field results of the antenna's 3D model are presented in Section III.

\section{Configuration of the Double-Layered RLSA ANTENNA}

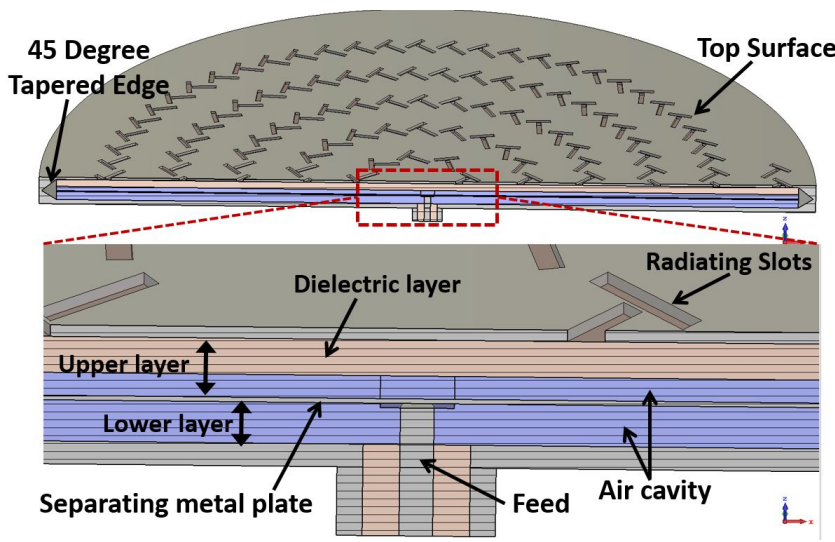

Fig. 1: Cross section view of the double layer radial line slot array antenna.

A cross section view of a double layer radial line slot array antenna is presented in Figure 1. Three parallel conducting plates are separated by a equal distance forming a two fold radial waveguide cavity. The top plate has a circular aperture and consisted with radiating slots. Slots on the top plate consist of many radiating slot pairs, each one of which is a unit radiator of a circularly polarised electric field vector. Slot pairs are arrayed in sequence along a long spiral ring. The radial slot spacing on the radiating plate was kept less than one guided wavelength to avoid grating lobes. Bottom plate works as a ground plane. A $0.2 \mathrm{~mm}$ thick copper plate is used in the middle of the waveguide to separate the upper and lower waveguide. Electromagnetic power is fed at the center of lower waveguide 
through a $50 \Omega$ coaxial cable and an outward travelling mode is generated. Upper layer of the waveguide is composed of dielectric material and air. Dielectric material is used to create a slow wave structure in the upper layer. Roger RT 5880 LZ (epsilon 1.96) was used as a dielectric material. The bottom layer of the waveguide is fully filled with air. At the outer edge of the waveguide a 45 Degree tapered edge was created. The tapered edge moves the outward travelling wave mode direction into inward travelling wave mode from the bottom layer to the upper layer, respectively. A $50 \Omega$ coaxial connector was inserted from the bottom of the lower waveguide. The coaxial pin of the connector is extended inside the air cavity of the bottom layer. PTFE was used as a coat of the connector.

\section{Simulation Results}

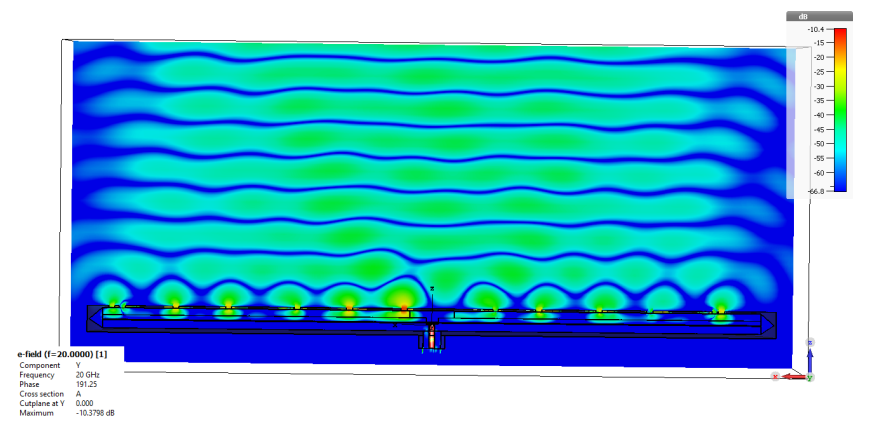

(a)

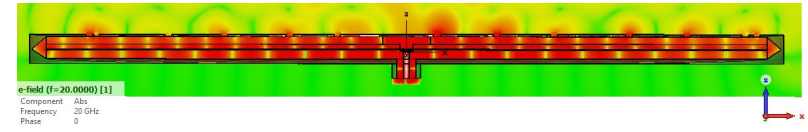

(b)

Fig. 2: Simulated electric near-field (a) Aperture illumination of $E_{y}$ in a cross section of $\mathrm{YZ}$ plane from the antenna aperture, (b) Wave propagation inside the cavity.

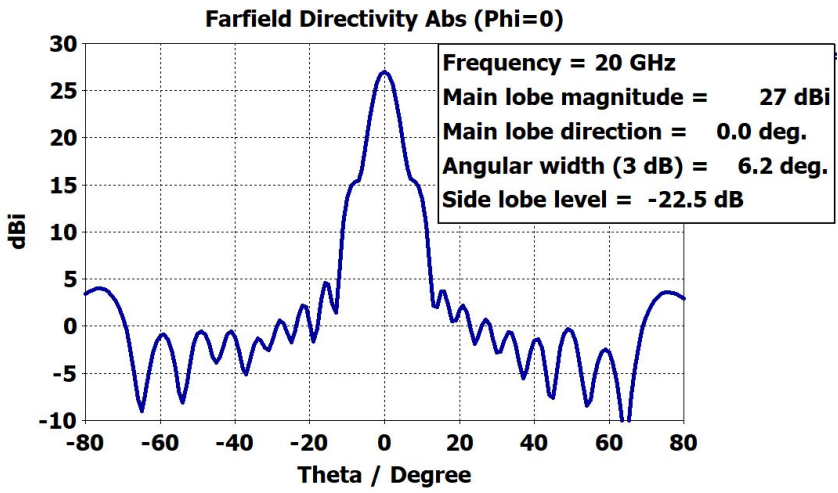

Fig. 3: Far-field radiation pattern cuts taken at $\phi=0$ degree plane at $20 \mathrm{GHz}$.

The antenna was simulated in time domain solver of CST Microwave Studio. The return loss performance of the antenna is reasonable and falls below $-10 \mathrm{~dB}$ in the operating frequency range. At $20 \mathrm{GHz}$, the antenna has shown a reflection coefficient of $-18.6 \mathrm{~dB}$. The electric near-field distribution of the antenna is shown in Figure 2. Figure(a) shows the illumination over the

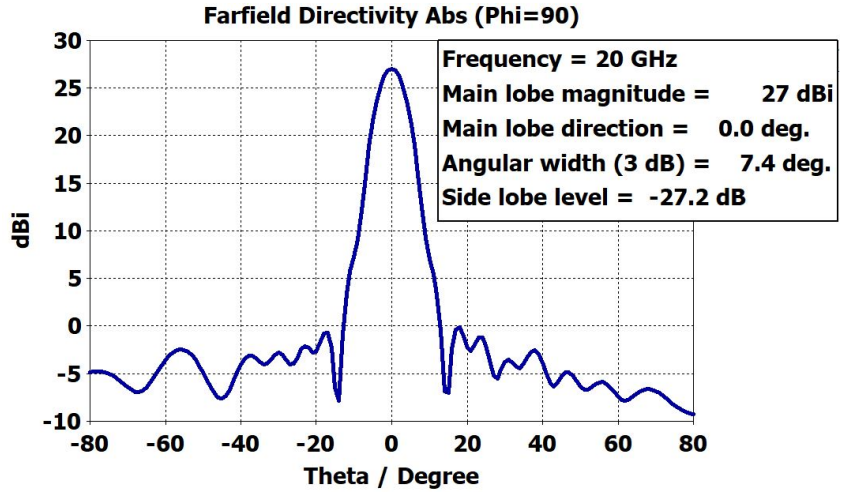

Fig. 4: Far-field radiation pattern cuts taken at $\phi=90$ degree plane at $20 \mathrm{GHz}$.

aperture of $E_{y}$ component in a cross section of $\mathrm{YZ}$ plane and figure (b) shows the wave propagation inside the waveguide cavity. It is evident from the near-field distribution that antenna has achieved a satisfactory uniform illumination over the aperture of the antenna. The antenna has demonstrated a peak directivity of $27.1 \mathrm{dBi}$ and a gain of $22.2 \mathrm{dBi}$ at $20 \mathrm{GHz}$. The difference between the directivity and gain is due to the reflections coming from boundary of the waveguide and energy losses in the upper layer of the waveguide. The radiation efficiency of the antenna has varied between (70-82)\% over the operating frequency range. The antenna has fulfilled the 3 -dB axial ratio condition of circular polarisation. The far-field radiation cut patterns taken at $\phi=0$ and $\phi=90$ degree plane at $20 \mathrm{GHz}$ are plotted in Figure 3 and Figure 4, respectively. In $\phi=0$ degree plane, the antenna has shown a side lobe level of $-22.5 \mathrm{~dB}$ with a $3-\mathrm{dB}$ angular beamwidth of 6.2 degree. In $\phi=90$ degree plane, the antenna has shown $-27.2 \mathrm{~dB}$ side lobe level with a $3-\mathrm{dB}$ angular beamwidth of 7.4 degree. In both plane the double layer slotted antenna has shown significantly lower side lobe levels providing good pattern quality.

\section{CONCLUSION}

In this paper we presented the design methodology of a double-layered radial line slot array (RLSA) antenna and their radiation performance. The antenna has demonstrated acceptable level of reflection coefficient and fulfilled the 3-dB axial ratio condition. This double layer circularly polarised RLSA antenna has provided uniform aperture illumination on the surface. The side lobe levels have significantly reduced which provide stable and good pattern quality.

\section{REFERENCES}

[1] M. Ando, K. Sakurai, N. Goto, K. Arimura, and Y. Ito, "A radial line slot antenna for $12 \mathrm{GHz}$ satellite TV reception," IEEE Transactions on Antennas and Propagation, vol. 33, no. 12, pp. 1347-1353, Dec 1985.

[2] P. W. Davis and M. E. Bialkowski, "Linearly polarized radial-line slotarray antennas with improved return-loss performance," IEEE Antennas and Propagation Magazine, vol. 41, no. 1, pp. 52-61, Feb 1999.

[3] N. Y. Koli, M. U. Afzal, K. P. Esselle, and M. Z. Islam, "Investigation on aperture field distribution of circularly polarised radial line slot array antennas," in 2018 IEEE Asia-Pacific Conference on Antennas and Propagation (APCAP), Aug 2018, pp. 462-463.

[4] M. U. Afzal and K. P. Esselle, "Steering the beam of medium-to-high gain antennas using near-field phase transformation," IEEE Transactions on Antennas and Propagation, vol. 65, no. 4, pp. 1680-1690, April 2017. 